\title{
BIOTEX—Biosensing Textiles for Personalised Healthcare Management
}

\author{
Shirley Coyle, King-Tong Lau, Niall Moyna, Donal O’Gorman, Dermot Diamond, Fabio Di Francesco, \\ Daniele Costanzo, Pietro Salvo, Maria Giovanna Trivella, Danilo Emilio De Rossi, Nicola Taccini, \\ Rita Paradiso, Jacque-André Porchet, Andrea Ridolfi, Jean Luprano, Cyril Chuzel, Thierry Lanier, \\ Frédéric Revol-Cavalier, Sébastien Schoumacker, Véronique Mourier, Isabelle Chartier, \\ Reynald Convert, Henri De-Moncuit, and Christina Bini
}

\begin{abstract}
Textile-based sensors offer an unobtrusive method of continually monitoring physiological parameters during daily activities. Chemical analysis of body fluids, noninvasively, is a novel and exciting area of personalized wearable healthcare systems. BIOTEX was an EU-funded project that aimed to develop textile sensors to measure physiological parameters and the chemical composition of body fluids, with a particular interest in sweat. A wearable sensing system has been developed that integrates a textile-based fluid handling system for sample collection and transport with a number of sensors including sodium, conductivity, and pH sensors. Sensors for sweat rate, ECG, respiration, and blood oxygenation were also developed. For the first time, it has been possible to monitor a number of physiological parameters together with sweat composition in real time. This has been carried out via a network of wearable sensors distributed around the body of a subject user. This has huge implications for the field of sports and human performance and opens a whole new field of research in the clinical setting.
\end{abstract}

Manuscript received February 15, 2009; revised July 22, 2009. First published January 8, 2010; current version published March 17, 2010. This work was supported by the European Union (Biotex FP6-2004-IST-NMP-2) and Science Foundation Ireland (07/CE/I1147).

S. Coyle, K.-T. Lau, N. Moyna, D. O'Gorman, and D. Diamond are with the CLARITY Centre for Sensor Web Technologies, Dublin City University, Dublin 9, Ireland (e-mail: shirley.coyle@dcu.ie; kim.lau@dcu.ie; niall.moyna@dcu.ie; donal.ogorman@dcu.ie; dermot.diamond@dcu.ie).

F. Di Francesco is with the Dipartimento di Chimica e Chimica Industriale, Università di Pisa, Pisa 56126, Italy (e-mail: fdifra@dcci.unipi.it).

D. Costanzo and M. G. Trivella are with the Istituto di Fisiologia Clinica, Consiglio Nazionale delle Ricerche, Pisa 56100, Italy (e-mail: costanzo.daniele@ libero.it; trivella@ifc.cnr.it).

P. Salvo and D. E. De Rossi are with the Centro Interdipartimentale di Ricerca “E. Piaggio," Università di Pisa 56100, Italy (e-mail: psalvo@ifc.cnr.it; d.derossi@ing.unipi.it).

N. Taccini and R. Paradiso are with Smartex s.r.1, Navacchio 56023, Italy (e-mail: Taccini@smartex.it; rita@smartex.it).

J.-A. Porchet, A. Ridolfi, and J. Luprano are with the Centre Suisse d'Electronique et de Microtechnique, CSEM SA, Neuchâtel CH-2007, Switzerland (e-mail: jacques-andre.porchet@csem.ch; andrea.ridolfi@csem.ch; jean.luprano@csem.ch).

C. Chuzel and T. Lanier are with Sofileta, Bourgoin-Jallieu 38300, France (e-mail: cyril.chuzel@sofileta.com; Thierry.lanier@sofileta.com).

F. Revol-Cavalier, S. Schoumacker, V. Mourier, and I. Chartier are with Electronics and Information Technology Laboratory of the French Atomic Energy Commission, MINATEC, Grenoble F38054, France (e-mail: jeanclaude. bouvier@thuasne.fr; sebastien.schoumacker@cea.fr; mounier.veronique@ cea.fr; ichartier@cea.fr).

R. Convert and H. De-Moncuit are with Thuasne, St. Etienne 42031, France (e-mail: reynald.convert@thuasne.fr).

C. Bini is with Penelope SpA, Montemurlo 59013, Italy (e-mail: cristina. bini@penelopespa.com).

Color versions of one or more of the figures in this paper are available online at http://ieeexplore.ieee.org.

Digital Object Identifier 10.1109/TITB.2009.2038484
Index Terms-Biomedical equipment, biomedical monitoring, intelligent materials, multisensor systems.

\section{INTRODUCTION}

W EARABLE sensors allow the continuous monitoring of a person's physiology in a natural setting. At the present stage, health-monitoring systems using electronic textiles are mainly targeting applications based upon physiological parameter measurements, such as body movements or ECG. To open a dramatically wider field of applications, chemical measurements on body fluids (blood, sweat, and urine) are needed. This area of research is unfortunately lacking due to the difficulty in sampling such fluids. The BIOTEX project has tackled some of these problems by developing a textile-based system to collect and analyze sweat by using a textile-based sensor capable of performing chemical measurements. The great advantage of analyzing sweat for health monitoring is that it is noninvasive, easily accessible, and it offers valuable physiological information [1], [2]. The sweat test is the gold standard technique for the diagnosis of cystic fibrosis (CF) [2]. This is a once-off test that is performed in newborns and the diagnosis is based on sodium and chloride concentration levels. However, advances in this direction have been limited due to the difficulty in obtaining uncontaminated samples. The BIOTEX system involves a network of textile sensors that can be easily integrated into everyday clothing and provide real-time physiological feedback.

During exercise, evaporation is usually the primary mechanism of heat dissipation. If the body cannot adequately evaporate sweat from the skin's surface, core temperature rises rapidly. A side effect of sweating is the loss of valuable fluids from the finite reservoir within the body. The sweat rate relates to exercise intensity, environmental conditions, acclimatization state, clothing, and baseline hydration status. It also varies between individuals. Sweat composition changes offer valuable information regarding hydration status and electrolyte balance and there are a number of fields where this can be applied [3], [4]. Hydration, performance, and physiological factors are all interrelated; therefore, by monitoring sweat loss and composition, in addition to heart rate and breathing, it is possible to get a more complete picture of the body's physiological state. Elite athletes must ensure electrolyte balance and adequate rehydration after exercise or risk-reduced performance [5]-[7]. Aside from athletes, dehydration can impact on everyone's general well-being, while in the elderly, dehydration can cause serious illness and 
even death, particularly, in the event of a heat wave [8]. Therefore, it is useful to measure the sweat loss and also to know the composition of sweat. Analysis of sweating patterns is also an important parameter, as this can indicate autonomic dysfunction. A straightforward method of sweat analysis could help in the diagnosis and treatment of various conditions that are affected by autonomic dysfunction, such as diabetes and hyperhydrosis [9], [10].

While sweat may be easily obtainable, collection and measurement techniques for analysis can be awkward-Minor's method involves covering the skin with starch-iodine powder that exhibits purple dots when sweat droplets appear [11]. "Wash-down" techniques involve exercising within a plastic enclosure to collect sweat and then washing down the body within the enclosure using deionized water at the end of the exercise trial [12]. These methods are obviously unsuitable for long-term measurements outside of a laboratory setting. Parafilm patches have been used to create a capsule on the skin surface, but these may change the sweat composition, as they prevent water evaporation [7]. Bioimpedance measurements have been used to estimate the amount of water in the body, but as a wearable system, this is challenging due to complex circuitry, measurement times, and the effects of movement and electrode placement [13], [14]. In the field, the most practical way for athletes to monitor their sweat loss is to measure changes in body weight pre and postexercise [15]. Therefore, it is clear that a method is needed to collect and analyze sweat in an unobtrusive way in normal settings, at home, in the gym, or on the track to provide real-time analysis of sweat activity.

This paper presents the design of a sweat analysis system that can be easily integrated into fabric for real-time analysis of sweat during exercise. A number of textile sensors are distributed around the body, including a multiparametric patch to measure the $\mathrm{pH}$, sodium concentration, and conductivity of sweat. The patch is responsible for collecting and delivering sweat samples to sensors and removing waste products. Other sensing modules include sweat rate, ECG, respiration, and blood oximetry. The design of each sensor is described and results of exercise trials are presented.

\section{METHODS}

The types of sensors that are integrated into the system and their placement on the body are listed in Table I. The sweat analysis sensors are positioned on the lower back using a waistband, while a separate garment holds ECG, respiration, and pulse oximetry sensors.

\section{A. Multiparametric Patch for Sweat Analysis}

1) Collection Methods/Fluidics: A textile-based platform with fluid handling properties is used to collect and analyze sweat samples. The system exhibits a passive pumping mechanism by capillary action using a combination of moisturewicking fabric and a highly absorbent material [16]. A textile channel is created using the moisture-wicking material while the absorbent is placed at the end of the channel. The absorbent controls fluid flow, thus drawing the sweat toward it along the length of the channel. In this way, a continuous flow of sweat
TABLE I

Wearable Sensors Integrated into TeXtiles and Sensor Placement

\begin{tabular}{|c|c|c|c|c|}
\hline & Parameter & $\begin{array}{l}\text { Sensing } \\
\text { Method }\end{array}$ & $\begin{array}{c}\text { Sensor } \\
\text { Placement }\end{array}$ & $\begin{array}{l}\text { Sensor } \\
\text { Range }\end{array}$ \\
\hline \multirow[t]{4}{*}{$\begin{array}{l}\text { Waist- } \\
\text { band }\end{array}$} & $\mathrm{pH}$ & Colorimetry & $\begin{array}{l}\text { Right } \\
\text { lower back }\end{array}$ & $\mathrm{pH} 4-8$ \\
\hline & Sodium & $\begin{array}{l}\text { Electro- } \\
\text { chemical }\end{array}$ & $\begin{array}{l}\text { Right } \\
\text { lower back }\end{array}$ & $10-100 \mathrm{nM}$ \\
\hline & Conductivity & Impedance & $\begin{array}{l}\text { Right } \\
\text { lower back }\end{array}$ & $2-15 \mathrm{mS} / \mathrm{cm}$ \\
\hline & Sweat rate & Capacitance & $\begin{array}{l}\text { Left lower } \\
\text { back }\end{array}$ & $\begin{array}{l}0.2-20 \\
\mathrm{~g} / \mathrm{m}^{2} \mathrm{~min}\end{array}$ \\
\hline \multirow{3}{*}{$\begin{array}{l}\text { Chest- } \\
\text { band/vest }\end{array}$} & ECG & Electrical & Thorax & $40-180 \mathrm{bpm}$ \\
\hline & Respiration & $\begin{array}{l}\text { Piezo- } \\
\text { resistivity }\end{array}$ & Thorax & $\begin{array}{l}12-40 \\
\text { breaths/min }\end{array}$ \\
\hline & $\begin{array}{l}\text { Pulse } \\
\text { oximetry }\end{array}$ & $\begin{array}{l}\text { Optical } \\
\text { Spectroscopy }\end{array}$ & Thorax & $80-100 \%$ \\
\hline
\end{tabular}

enters the textile-based channel where it is analyzed by the sensors, and then, it travels toward the absorbent where it is stored. The channel is formed by screen-printing a hydrophobic material on either side of a polyester/lycra blend. Following this, a polyurethane film is placed on the skin side of the fabric, thus leaving a small area at the top of the channel uncovered. This forms the inlet through which the sweat enters the pump. The dimensions of the channel have a large influence on the rate of fluid flow. The rate increased for wider channel width and decreased for longer channel lengths. Therefore, the channel can be designed in order to collect sweat at a speed that matches the sweat rate of the human body. The dimensions of the patch were based on sweat rate measurements by Patterson et al. [17]. The forehead, chest, and lower back are regions that generate the most amount of sweat. The lower back was chosen, as this is an unobtrusive location for sensor placement during exercise. The sweat rate on the lower back is reported to be $0.85 \pm 0.41 \mathrm{mg} / \mathrm{cm}^{2}$ [17]. The channel design is shown in Fig. 1. In order to maximize the sweat collection over the area covered by the textile patch and move it toward the inlet to the channel, a fabric with a triple layer of materials (Coolmax/ polyester/polyester) is stitched to the skin side of the patch, as shown in Fig. 1(b). The patch collects sweat from an area of $22 \mathrm{~cm}^{2}$; therefore, the expected flow rate of the pump is $17 \mathrm{mg} / \mathrm{min}$. A small piece $(1 \mathrm{~cm} \times 3 \mathrm{~cm})$ of superabsorbent (Absorbtex) with a free swell capacity $25.1 \mathrm{~g} / \mathrm{g}$ is used, which means that the pump should operate for $75 \mathrm{~min}$, assuming a constant sweat loss rate of $17 \mathrm{mg} / \mathrm{min}$.

2) pH Sensor: A colorimetric approach using $\mathrm{pH}$ sensitive dyes has been used to develop a textile-based $\mathrm{pH}$ sensor. Such dyes have different absorption properties depending on their immediate $\mathrm{pH}$ environment. The $\mathrm{pH}$ indicator is immobilized onto the textile substrate and the color is monitored using optical components arranged in a reflectance-mode configuration shown in Fig. 2. The dye used is bromocresol purple (BCP) that responds to the $\mathrm{pH}$ range $4-7$ and tetraoctyl ammonium bromide is used to immobilize the dye [18], [19]. For optical detection, a pair of LEDs is used. LEDs have been demonstrated to operate as detectors as well as light sources and offer a low-cost 


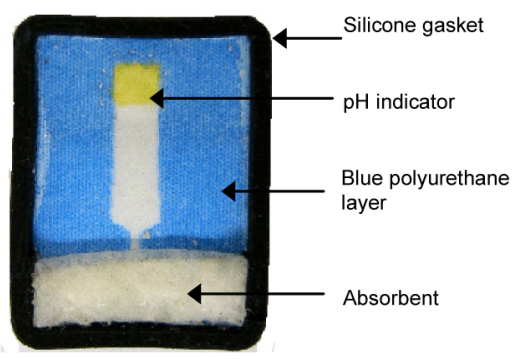

(a)

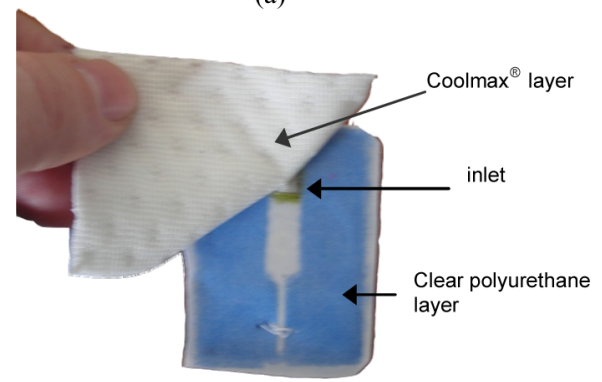

(b)

Fig. 1. Textile-based fluid handling platform.

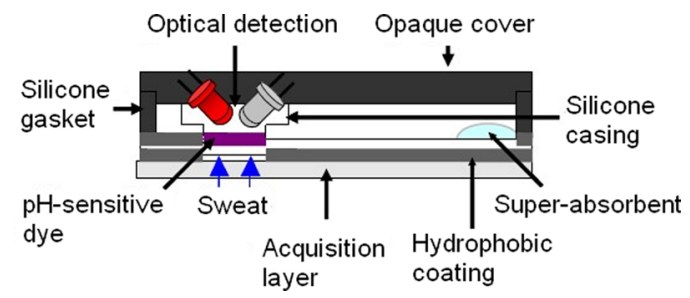

Fig. 2. pH sensor configuration.

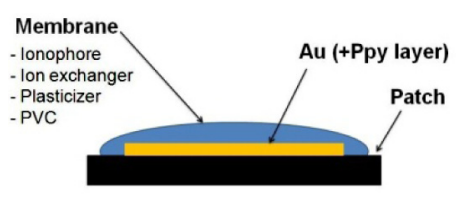

(a)

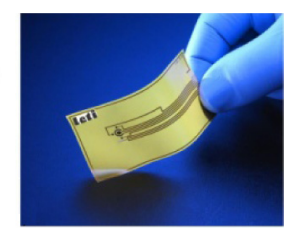

(b)
Fig. 3. (a) Schematic representation of the working electrode layer $\left(\mathrm{Na}^{+}\right.$ISE). (b) Picture of the electrochemical cell on flexible film.

and low-power solution, which is desirable for any wearable application.

3) Sodium Sensor: The sodium sensor, developed by Electronics and Information Technology Laboratory of the French Atomic Energy Commission (CEA-LETI), is fabricated on a flexible kapton surface. The principle of the electrochemical sensor consists of the measurement of the open-circuit potential (OCP) between a reference gold electrode and an ion-selective electrode (solid contact ion-selective electrode, SC-ISE), which is a function of the sodium concentration. This $\mathrm{Na}^{+}$selective electrode is made on a gold contact covered with a polymeric membrane that contains a polymer (polypyrrole, PPy), plasticizer, ionophore, and ion exchanger, as shown in Fig. 3.

The polymeric ion-selective membrane requires a conditioning period before use $(12 \mathrm{~h}$ in $1 \mathrm{mM} \mathrm{NaCl})$ and must be cal-

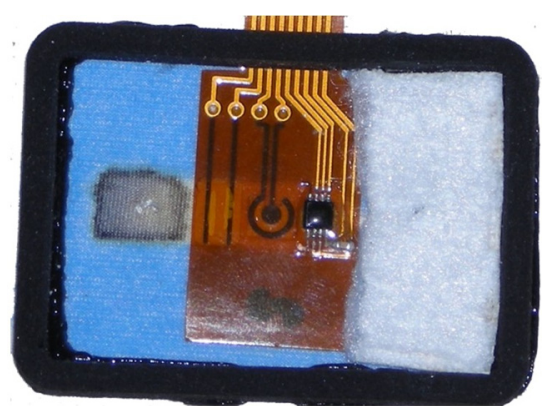

Fig. 4. Multiparametric patch containing $\mathrm{pH}$ indicator, conductivity, sodium, and temperature sensors.

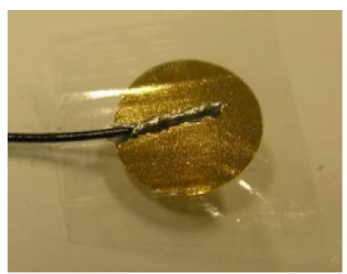

(a)

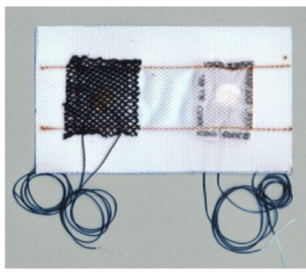

(b)
Fig. 5. (a) Wearable humidity sensor. (b) Textile arrangement to measure low sweat rate values.

ibrated prior to the trials, using model solutions with known concentrations of sodium $(20,40,60$, and $80 \mathrm{mM})$. This sodium electrochemical sensor is reusable and requires only one step of washing before reuse.

a) Conductivity sensor: The conductivity sensor consists of electrodes fabricated on the flexible kapton surface that also contains the sodium sensor. As conductivity and sodium measurements are temperature dependent, a temperature sensor (Analog Device ADT7301) is included within the system to compensate for temperature changes. The kapton patch is placed across fluidic channel, as shown in Fig. 4. Literature reports a range of values from 2 to $15 \mathrm{mS} / \mathrm{cm}$, with an average conductivity of $5 \mathrm{mS} / \mathrm{cm}$ for the sweat of healthy subjects [20].

b) Sweat rate sensor: The skin is a complex structure, but to model perspiration it can be effectively approximated by a homogenous flat surface continuously emitting water vapor. With such an assumption, Fick's first law of diffusion can be used to calculate sweat rate from the gradient of humidity measured by a pair of wearable humidity sensors at two distances $(0.5$ and $1.5 \mathrm{~cm})$ from the skin. In cases of low sweat rates $\left(10-40 \mathrm{~g} /\left(\mathrm{m}^{2} \cdot \mathrm{h}\right)\right)$, more accurate estimates can be obtained by increasing the gradient by means of a net fabric ( $88 \%$ polyamide and $12 \%$ elastane). Fig. 5 shows a humidity sensor and the latter textile arrangement. The textile membrane is removed when the measurement range has to be extended up to $1000 \mathrm{~g} /\left(\mathrm{m}^{2} \cdot \mathrm{h}\right)$. In that case, a flexible textile frame is used to place the sensors at the right positions. Capacitive humidity sensors are prepared either by vacuum-depositing gold electrodes onto hydrophilic films (polyvinyl alcohol or cellulose acetate butyrate) or by modifying a commercial device (Philips H1). 


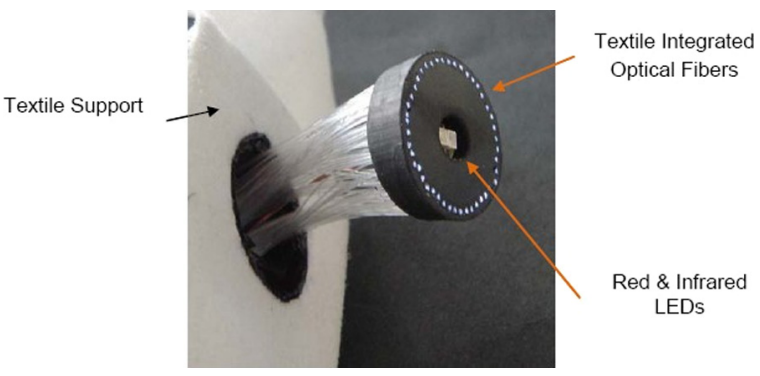

Fig. 6. Thoracic oximetry sensor located at the upper sternum.

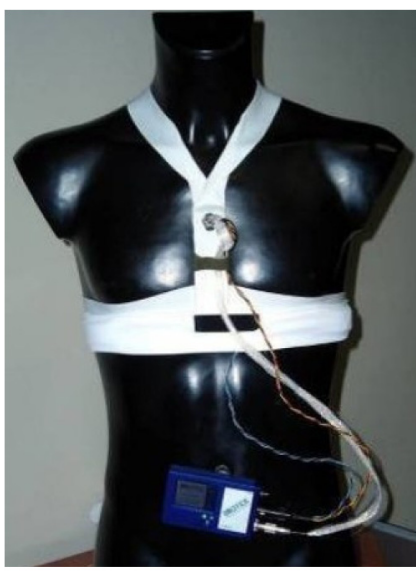

(a)

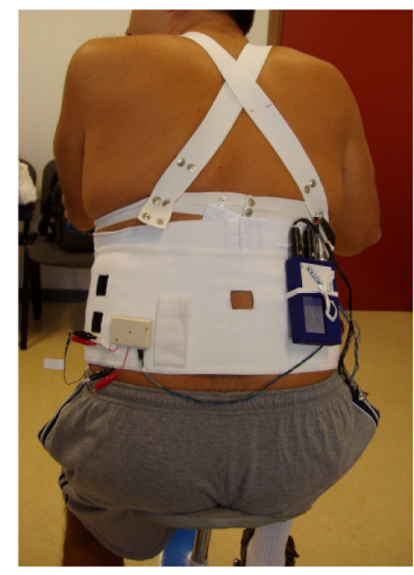

(b)
Fig. 7. (a) Arrangement of the ECG, respiration, and thoracic oximetry sensors. (b) Experimental setup during an exercise trial.

\section{B. Chest band/Vest}

1) Thoracic Oximetry Sensor: Commercial pulse oximeters measure blood oxygenation $\left(\mathrm{SpO}_{2}\right)$ at the fingertip or earlobe. Such sites benefit from a dense vascular bed providing highquality information on blood oxygenation. Nevertheless, they are not textile accessible and fingertip or earlobe $\mathrm{SpO}_{2}$ probes are quite obtrusive, as they interfere with the user's daily activities. The BIOTEX $\mathrm{SpO}_{2}$ sensor measures blood oxygenation at the sternum. The sensor is shown in Fig. 6. At its centre, the circular plastic support holds red and infrared LEDs that emit light to the sternum. At the outer contour, the optical fibers are positioned to receive the light reflected at the sternum.

Optical fibers integrated in the fabric make it possible to access the sternum while ensuring user comfort. It is possible to produce a bus with a high number of fibers. An elastic version of the optical fiber fabric further improves comfort [21]. A textile shoulder strap has been designed and realized to keep the optical system in place while also applying the right pressure to the skin [see Fig. 7(a)].

2) ECG and Respiration: Three textile electrodes with silicone cushions, to improve signal stability, are integrated into the garment to measure ECG signals. The use of a seamless knitting-dedicated machine (i.e., Santoni SpA5) provides elastic, adherent, and comfortable garments. The devices are realized using stainless-steel yarns produced by Bekintex for the electrodes and the Meryl Skinlife purchased by Nylstar as basal
TABLE II

Signal ACQUisition AND SAMPLING FREQUENCIES

\begin{tabular}{ll}
\hline \hline \multicolumn{1}{c}{ Signal } & \multicolumn{1}{c}{ Sampling frequency } \\
\hline Electrocardiogram (ECG) & $250 \mathrm{~Hz}$ \\
Pulsed oximetry & $250 \mathrm{~Hz}$ \\
Colorimetric pH sensing & $0.2 \mathrm{~Hz}$ \\
Respiration plethysmography & $25 \mathrm{~Hz}$ \\
Chest-band/vest & $0.5 \mathrm{~Hz}$ \\
Electrochemical sensors Na+ & $1 \mathrm{~Hz}$ \\
Sweat conductivity (SWCO) & $0.2 \mathrm{~Hz}$ \\
Sweat rate (SWRA) & $0.2 \mathrm{~Hz}$ \\
Sweat temperature & $0.2 \mathrm{~Hz}$ \\
\hline \hline
\end{tabular}

yarn. A piezoresistive sensor responds to the movement of the ribcage during breathing and measures respiration rate.

3) Control Electronics: A central electronics unit powers controls and saves data from each of the sensors. Data are either transmitted over Bluetooth for remote saving and further processing or saved by an SD card recorder included in the control electronics. A touch-screen display is used to set the configuration parameters as a simple user interface. This allows the user to navigate through the menus and to view the results processed by the unit. Graphical presentation on a laptop is also used to simplify the interpretation of the data. Table II describes the measurements carried out by the control electronics. The control electronic also extracts the heart rate and the $\mathrm{SpO}_{2}$ from the measured signals.

\section{EXPERIMENTAL SETUP}

In order to test the sensors and fabric patch on-body, the sweat analysis sensors are enclosed in a waistband, designed by Smartex, which ensures that the fabric patch maintains good contact with the skin. It is also used to reduce motion artefacts during exercise and block ambient light, which may affect the operation of the optical-detection unit of the $\mathrm{pH}$ sensor. The waistband is made from a moisture wicking, stretch fabric for comfort with an integrated pocket to hold the multisensor patch in place. The use of an elastic fabric laminated with a hydrophobic membrane prevents the absorption of sweat from the waistband, which would reduce the sweat flow to the pump. Hooks and loops fasteners are used to keep the passive pump and the electronics in place, thereby reducing motion effects during exercise.

A second pocket houses the sweat rate sensor. A neoprene frame makes sure that the right distance between sensors and skin is maintained, while holes in the pocket allow the water vapor to diffuse away without obstacles.

The waistband is positioned on the subject's back, as shown in Fig. 7(b). The multiparametric patch is to the right of the spine, while the sweat rate sensor is positioned to the left of the spine. This is done to avoid collecting sweat, which runs from the top to the bottom of the back along this hollow.

\section{RESULTS}

Exercise trials involved indoor cycling at $20^{\circ} \mathrm{C}$ for up to $60 \mathrm{~min}$. Subjects typically started sweating after 5-10 min. 


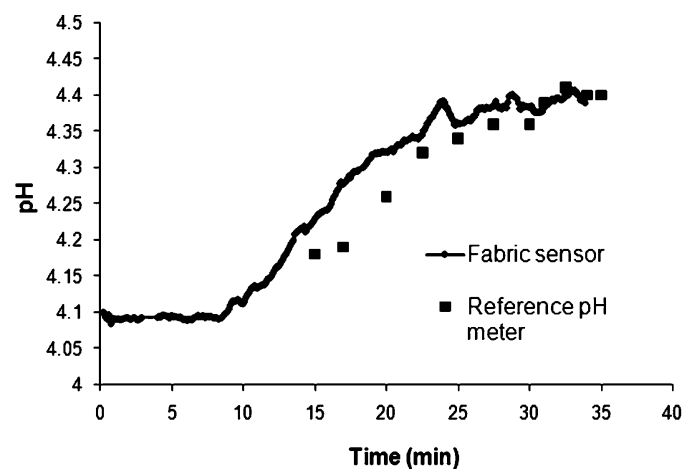

Fig. 8. Sweat $\mathrm{pH}$ during an exercise trial.

\section{A. Fabric Fluid Handling Platform}

Fluid transportation is achieved through capillary action in a moisture-wicking fabric in conjunction with a superabsorbent material. In this way, a continuous flow of sweat may enter the textile-based channel, where it is analyzed by the sensors and then travels on to the absorbent where it is stored. The weight of the absorbent on the fabric patch was recorded before and after exercise, in order to obtain an estimate of the flow rate of sweat through the textile patch. Sweat rates ranged from 4 to $8.3 \mathrm{mg} / \mathrm{min}$. Female subjects tended to have lower sweat rates compared to males, which is well established [22]. Lower sweat rate affects the time for sweat to reach the sensors, and if the flow rate is too low, there is not adequate fluid to wet the sensors on the kapton layer that touch the surface of the fabric channel.

\section{B. pH Sensor}

The ability of the textile-based $\mathrm{pH}$ sensor to measure real-time changes in sweat $\mathrm{pH}$ during exercise was assessed by comparing the results of the sensor to those obtained using a Skincheck $\mathrm{pH}$ meter. This is a hand-held device used to measure skin surface $\mathrm{pH}$. Reference measurements were taken from the fabric surface of a reference fabric patch positioned beside the multiparametric patch. The reference device is not wearable and does not allow for continuous real-time measurements. Point measurements were taken every 2 min once there was sufficient sweat on the fabric patch, i.e., after $15 \mathrm{~min}$ during the trial depicted in Fig. 8. The wireless fabric sensor data shows close correlation with the handheld reference meter (mean absolute error $=0.03 \mathrm{pH}$ units).

\section{Sodium Sensor and Conductivity Sensor}

The responses of the sodium and conductivity sensors are shown in Fig. 9. Meaningful measurements can be taken once sufficient sweat begins to enter the channel and makes contact with the sensors. Following this priming period, the electrodes respond to the sweat on the surface of the fabric channel. For the trial depicted in Fig. 9, this takes approximately $35 \mathrm{~min}$. After this time, the sodium sensor reaches a steady-state value corresponding to a concentration of $15 \mathrm{mM}$. The duration of the priming period is not constant among different subjects, as it depends on how much and how fast the subject is sweating.
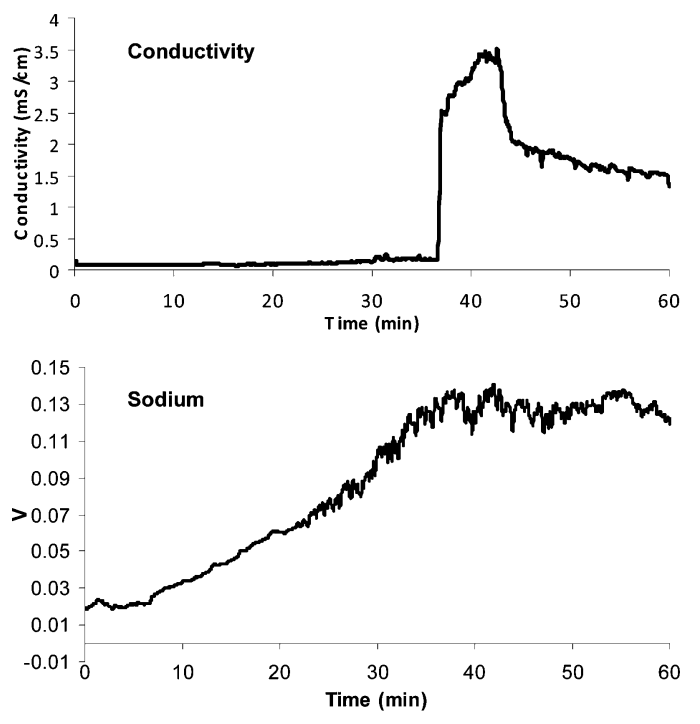

Fig. 9. Response of sodium and conductivity sensors during an exercise trial.

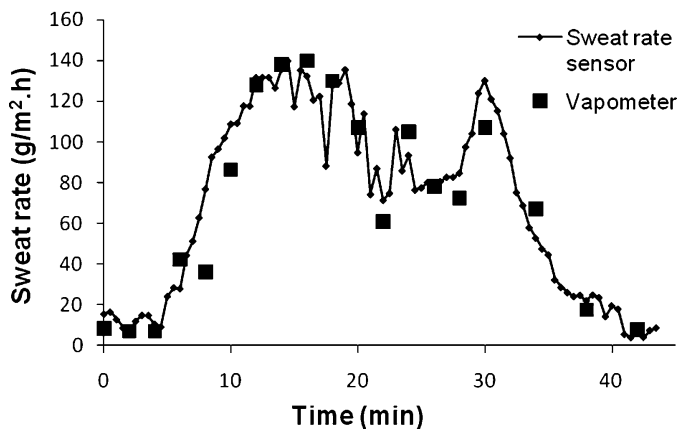

Fig. 10. Textile sweat rate sensor compared to commercial VapoMeter.

The signal from the sweat rate sensor can give an indication of when there is sufficient flow of sweat to the sensors for the measurements to be valid.

\section{Sweat Rate Sensor}

Sensor development and calibration is described by Salvo et al. [23]. The difference in capacitance between the bottom and top humidity sensors has been considered as an indication of sweat rate. The results of a trial are shown in Fig. 10. The trial lasted 45 min and involved 30 min of indoor cycling, at a selfselected pace, following by $15 \mathrm{~min}$ rest. A commercial meter (VapoMeter, Delphintech) for measuring water evaporation was used as a reference, as there are currently no wearable devices available to measure sweat rate in real time. The VapoMeter is a hand-held device, but it is not wearable and needs about $2 \mathrm{~min}$ to perform a point measurement. From the results of the trial, it can be seen that the subject started sweating after approximately $5 \mathrm{~min}$ and that sweat rate declined as soon as the subject rested after $30 \mathrm{~min}$. The measurements taken by the textile sensor are congruent with those provided by the VapoMeter (mean absolute error $\left.=10.17 \mathrm{~g} /\left(\mathrm{m}^{2} \cdot \mathrm{h}\right)\right)$. 
TABLE III

PERFUSION INDEX FOR THE RED AND INFRARED LIGHT AT THE FINGER, THE FOREHEAD, AND THE STERNUM

\begin{tabular}{ccc}
\hline \hline Location & $\begin{array}{c}\text { Perfusion index } \% \\
\text { Red light }\end{array}$ & $\begin{array}{c}\text { Perfusion index } \% \\
\text { Infrared light }\end{array}$ \\
\hline Finger & 2.92 & 5.09 \\
Forehead & 0.57 & 1.16 \\
Chest & 0.13 & 0.18 \\
\hline \hline
\end{tabular}

\section{E. ECG and Respiration}

The textile sensors to measure ECG and respiration are based on the knowledge gained from previous EU projects including MyHeart and Wealthy [24]. The applied sensing technology has been tested against gold standard references in previous work [25]. The signal-to-noise ratio has been high enough for all the tests, in order to make it possible to compute heart rate with the internal algorithm of the BIOTEX control electronics. Signals gathered during rest show high quality and reliability allowing the study of the $\mathrm{T}$ wave and morphology analysis.

The goal of the breathing measurement system was to measure the respiration rate, which has been achieved. In two subjects who had very high sweat rates, the signal showed significant drift and noise. The quantity of sweat that soaked the band caused a variation of the electrical resistance of the sensor. This variation was simply reduced by applying a bandpass filter of $0.2-2 \mathrm{~Hz}$.

\section{F. Thoracic Oximetry}

The finger is generally the preferred location for measuring oxygen saturation. Table III shows the perfusion index of the finger compared to the forehead or chest. The finger or forehead may prove to be obtrusive during exercise, whereas measuring oxygen saturation on the chest by a sensor embedded in a t-shirt is more appealing solution. Comfort, practicality, and appearance are issues that must be addressed when designing wearable sensing devices. As shown by the figures in Table III, this unobtrusive placement comes at the price of a much lower perfusion index, compared to the fingertip, which means that the detection of reflected light is more challenging. Motion artifacts during physical activity have added to this challenge, while sweat on the skin surface also affects the authenticity of the signal.

A BIOPAC fingertip $\mathrm{SpO}_{2}$ probe has been used to provide a reference measurement. Blood oxygenation variations have been induced by causing hypoxic periods using a high-altitude simulator (Altitrainer4). Fig. 11 shows the response of the BIOTEX sternum sensor with the reference fingertip probe measurement while the subject was seated.

\section{DISCUSSION}

Further testing is needed to establish the reasons for the changing composition of sweat during exercise, as research in this area is unfortunately lacking due to the difficulty in sampling sweat. Changes in sweat composition during exercise may be explained by considering the anatomy of sweat glands and their operation. Sweat glands have two main components, a

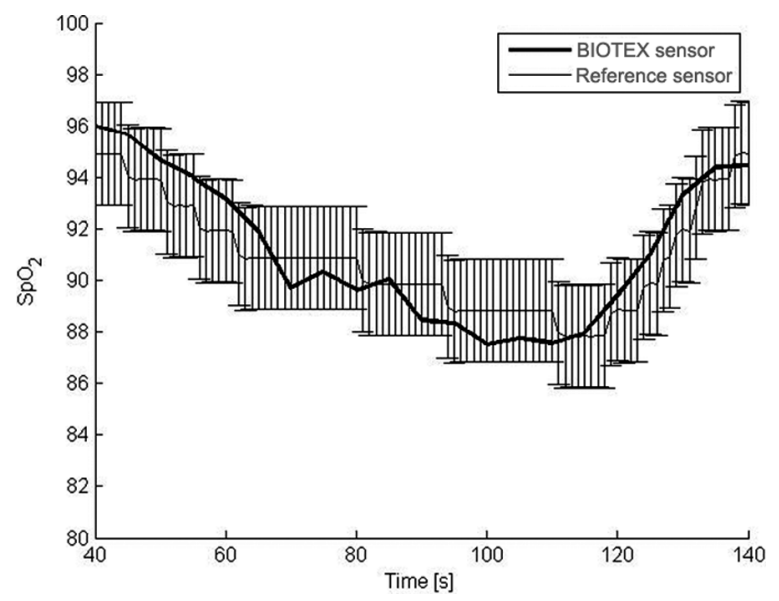

Fig. 11. BIOTEX $\mathrm{SpO}_{2}$ sensor compared with the reference measurement.

tubular coiled area where the sweat is produced and the duct through which it reaches the skin surface. The secretory portion of the eccrine sweat gland produces an isotonic fluid that moves through the ductal portion of the sweat gland, where solutes such as sodium ions are reabsorbed. One hypothesis is that during exercise, the sweat secreted at the skin is initially acidic due to reabsorption processes in the sweat duct. During exercise, an increase in sweat rate affects the reabsorption processes in the sweat duct and increases the electrolyte concentration and $\mathrm{pH}$ of the sweat on the skin surface [26]. During an exercise trial, the sweat secreted at the skin is initially acidic due to these reabsorption processes that occur in the duct. However, as the subject continues to exercise, the rate at which sweat is excreted increases, in order to regulate body temperature. This reduces the time available for reabsorption processes, and therefore, sweat $\mathrm{pH}$ increases along with sodium concentration and conductivity. Further work is needed to investigate the changes in sweat compositions during different training regimes in different environmental conditions.

With the current design, the sensors responded well provided that there was an adequate supply of sweat. While this is not a problem for fit healthy subjects, the design needs to be adapted for users who may not be capable of strenuous physical exercise. Reducing sensor size and thereby reducing the channel dimensions would help to improve this. This would also help to reduce the priming time involved. Indeed, in the case of sodium and conductivity sensors, the trial results showed that if good contact between the sensors and the textile channel can be maintained, it is possible to make measurements in real time. A future improvement may be to change the fabric or the design of the textile-based channel, in order to improve the absorption of sweat.

\section{CONCLUSION}

The textile pump has been designed in such a way that it can successfully collect sweat from human subjects during exercise. It then moves that sweat along a predefined channel, where it is analyzed by $\mathrm{pH}$, sodium, and conductivity sensors and stores the sweat in an absorbent in such a way as to allow 
for a continuous flow of fresh sweat. Therefore, a passive pumping mechanism has been realized requiring no external power supply. Real-time information regarding sweat composition has been measured during exercise. A sweat rate sensor was also developed as a separate device. In addition to chemical-sensing measurements, other physiological parameters have been measured by developing a complete network of textile-embedded sensors. Ultimately, these would be distributed within the one garment providing complementary information to give an overall picture of the wearer's health status.

Changes in the composition of sweat can be used to provide information on a person's well-being and physiological condition [3], [4], [6]. In the case of athletes who partake in endurance sports analysis of sweat can give information on hydration levels. Dehydration affects performance and if left untreated can lead to symptoms such as irritability, headache, dizziness, cramps, vomiting, increased body temperature and heart rate, increased perceived work rate, reduced mental function, and slower gastric emptying. These symptoms can be avoided and a high level of performance is maintained if the correct amount of fluids and $\mathrm{Na}^{+}$are ingested.

Endurance sports are not just the domain of the elite athlete. It is becoming increasingly popular for people to train for events such as the half and full marathon. In addition to the risks of drinking too little, ingesting too much water can lead to hyponatremia, which is characterized by low levels of sodium. Where there is a quick onset of hyponatremia, for example, during prolonged exercise, it can lead to severe complications such as seizures, coma, brain damage, and death.

The ability to measure changes in sweat electrolyte concentrations can assist people in choosing the correct level of hydration and avoid the need for medical intervention. It may also be used to protect amateur sports people from developing potentially fatal conditions.

The advantages of noninvasive monitoring of body fluids such as sweat has been touched in this paper, but further investigation is needed to explore this new and exciting area of research. The textile sensor system provides a straightforward, real-time measurement system, which may prove to be a valuable tool for medical research, and particularly, sports physiology. The feasibility of real-time and continuous chemical monitoring in a garment has been demonstrated. BIOTEX forms part of a textile roadmap, which has included projects such as MyHeart and WEALTHY in the past and currently PROETEX, aiming to provide health-monitoring tools through textiles to bring the benefits of safety and comfort to the users.

\section{REFERENCES}

[1] A. G. R. Whitehouse, "The dissolved constituents of human sweat," Proc. R. Soc. Lond., vol. 803, pp. 139-154, 1935.

[2] J. Massie, K. Gaskin, P. Van Asperen, and B. Wilcken, "Sweat testing following newborn screening for cystic fibrosis," Pediatr. Pulmonol., vol. 29 , pp. 452-456, 2000.

[3] J. Weber, A. Kumar, and S. Bhansali, "Novel lactate and pH biosensor for skin and sweat analysis based on single walled carbon nanotubes," Sens. Actuators B, vol. 117, pp. 308-313, 2006.
[4] R. M. Morgan, M. J. Patterson, and M. A. Nimmo, "Acute effects of dehydration on sweat composition in men during prolonged exercise in the heat," Acta Physiol. Scand., vol. 182, pp. 37-43, 2004.

[5] S. J. Montain, "Hydration recommendations for sport 2008," Curr. Sports Med. Rep., vol. 7, pp. 187-192, 2008.

[6] R. J. Maughan, J. B. Leiper, and S. M. Shirreffs, "Rehydration and recovery after exercise," Sports Sci. Exch., vol. 9, no. 62, pp. 1-5, 1996.

[7] S. M. Shirreffs and R. J. Maughan, "Whole body sweat collection in humans: An improved method with preliminary data on electrolyte content," J. Appl. Physiol., vol. 82, pp. 336-341, 1997.

[8] M. Olde Rikkert, R. Melis, and J. Claasen, "Heat waves and dehydration in the elderly," BMJ, vol. 339, pp. 119-120, 2009.

[9] M. Asahina, Y. Yamanaka, Y. Akaogi, S. Kuwabara, Y. Koyama, and T. Hattori, "Measurements of sweat response and skin vasomotor reflex for assessment of autonomic dysfunction in patients with diabetes," $J$. Diabetes Complications, vol. 22, pp. 278-83, 2008.

[10] A. Haider and N. Solish, "Focal hyperhidrosis: Diagnosis and management," Can. Med. Assoc. J., vol. 172, pp. 69-75, 2005.

[11] K. T. Sato, A. Richardson, D. E. Timm, and K. Sato, "One-step iodine starch method for direct visualization of sweating," Amer. J. Med. Sci., vol. 6, pp. 528-531, 1988.

[12] P. W. Lemon and K. E. Yarasheski, "Feasibility of sweat collection by whole body washdown in moderate to high humidity environments," Int. J. Sports Med., vol. 6, pp. 41-43, 1985.

[13] T. Vuorela, K. Kuttonen, J. Rantanen, T. Jaervinen, and J. Vanhala, "Bioimpedance measurement system for smart clothing," in Proc. 7th IEEE Int. Symp. Wearable Comput., 2003, pp. 98-107.

[14] G. Medrano, L. Beckmann, N. Zimmermann, T. Grundmann6, T. Gries, and S. Leonhardt, "Bioimpedance spectroscopy with textile electrodes for a continuous monitoring application," presented at the 4th Int. Workshop Wearable Implantable Body Sens. Netw. (BSN 2007), Mar. 26-28, RWTH Aachen University, Germany.

[15] R. J. Maughan and S. M. Shirreffs, "Development of individual hydration strategies for athletes," Int. J. Sport Nutr. Exerc. Metab., vol. 18, pp. 457$472,2008$.

[16] G. Wallace, D. Diamond, K. Lau, S. Coyle, Y. Wu, and D. Morris, "Flow analysis apparatus and method," U.S. Patent Application No. PCT/EP2008/051423, Aug. 14, 2008.

[17] M. Patterson, S. Galloway, and M. A. Nimmo, "Variations in regional sweat composition in normal human males," Exp. Physiol., vol. 85, pp. 869-876, 2000 .

[18] S. Coyle, Y. Wu, K. Lau, S. Brady, G. Wallace, and D. Diamond, "Biosensing textiles-Wearable chemical/bio-sensors for health monitoring," presented at the 4th Int. Workshop Wearable Implantable Body Sens. Netw., Aachen, Germany, 2007.

[19] D. Morris, S. Coyle, Y. Wu, K. T. Lau, G. Wallace, and D. Diamond, "Bio-sensing textile based patch with integrated optical detection system for sweat monitoring," Sens. Actuators B, vol. 139, pp. 231-236, 2009.

[20] T. S. Licht, M. Stern, and H. Shwachman, "Measurement of the electrical conductivity of sweat," Clin. Chem., vol. 3, pp. 37-48, 1957.

[21] G. Marcello, "Textile product with illuminated fibres, article made there from and production method of the same," U.S. Patent Application 20030156426, Aug. 21, 2003.

[22] C. H. Wyndham, J. F. Morrison, and C. G. Williams, "Heat reactions of male and female Caucasians," J. Appl. Physiol., vol. 20, pp. 357-364, 1965.

[23] P. Salvo, F. Di Francesco, D. Costanzo, C. Ferrari, M. G. Trivella, D. De Rossi, "A wearable sensor for measuring sweat rate," IEEE Sensors J. Lett., to be published.

[24] R. Paradiso and D. De Rossi, "Advances in textile technologies for unobtrusive monitoring of vital parameters and movements," in Proc. 28th IEEE EMBS Annu. Int. Conf., New York City, 2006, pp. 392-395.

[25] R. Paradiso, G. Loriga, and N. Taccini, "A wearable health care system based on knitted integrated sensors," IEEE Trans. Inf. Technol. Biomed., vol. 9, no. 3, pp. 337-344, Sep. 2005.

[26] E. Fishberg and W. Bierman, "Acid base balance of sweat," J. Biolog. Chem., vol. 97, pp. 433-441, 1932.

Author's photographs and biographies not available at the time of publication. 